\title{
IDENTIFICACIÓN DE TRAUMA URETERAL EN UN HOSPITAL DE TERCER NIVEL*
}

\author{
Drs. Herney Andrés García-Perdomo ${ }^{1}$, Ricardo Contreras G. ${ }^{1}$, Diego Castillo C. ${ }^{1}$ \\ 1 Servicio de Urología. Facultad de Salud. Escuela de Medicina. Universidad del Valle. \\ Cali, Colombia.
}

\begin{abstract}
\section{Ureteral trauma. Report of five cases}

Introduction: Ureteral trauma can result from various noxas the most frequent iatrogenic. Additional this, external causes: blunt trauma, sharp weapon and gunshot contributing to the overall incidence. Its early diagnosis although great importance to avoid complications is difficult so it is their suspect is essential when confronted with trauma patients. Case reports: We report five patients aged 15 to 46 years with ureteral trauma. Two patients had gross hematuria, one patient acute abdominal pain and two as hypovolemic shock. The lesions were found mainly to the left ureter and in smaller proportion in right ureter. Four patients the diagnosis was surgical exploration and one case was performed by scans. All were operated, had an uneventful postoperative period and are currently asymptomatic.
\end{abstract}

Key words: Wounds and injuries, ureteral injury, complications.

\section{Resumen}

Introducción: El trauma de uréter puede ser resultado de diferentes noxas siendo la más frecuente la iatrogénica. Adicional a esto, las causas externas de trauma contuso, las causadas con arma cortopunzante y las realizadas por arma de fuego contribuyen a la incidencia global. Su diagnóstico temprano, aunque de gran importancia para evitar complicaciones, resulta difícil por lo que es primordial su sospecha cuando nos enfrentamos a pacientes politraumatizados. Casos clínicos: Se identificaron 5 casos de trauma ureteral en nuestro hospital desde septiembre de 2012 a agosto de 2013. La mediana de edad fue 24,5 años (15-46 años). Se manifestaron como hematuria macroscópica en dos casos, un caso como dolor abdominal agudo y dos como choque hipovolémico. Estas lesiones se encontraron principalmente localizadas hacia uréter izquierdo y en menor proporción en uréter derecho, todas de tipo penetrante, causadas por arma de fuego. En 4 pacientes el diagnóstico fue por exploración quirúrgica y un caso fue realizado a través de escanografía. Los pacientes fueron intervenidos quirúrgicamente, ninguno presentó complicaciones y se encuentran actualmente asintomáticos.

Palabras clave: Heridas y lesiones, trauma de uréter, complicaciones.

*Recibido el 8 de septiembre de 2014 y aceptado para publicación el 4 de octubre de 2014.

Los autores declaramos que no hay conflicto de interés y no se recibieron fondos para el desarrollo de la presente investigación.

Correspondencia: Dr. Herney García-Perdomo

Herney.garcia@correounivalle.edu.co 


\section{Introducción}

La incidencia de trauma abdominal se ha incrementado en las últimas décadas como consecuencia de los accidentes vehiculares y de la violencia que azota a nuestro país ${ }^{1}$, pudiendo llegar a representar entre 15 a $20 \%$ de todas las muertes por trauma. Esas muertes pueden ocurrir de forma temprana como resultado de hemorragia o posteriormente como una complicación de la sepsis ${ }^{2}$. En relación al trauma genitourinario, es a menudo pasado por alto en casos de trauma agudo debido a que otras lesiones que amenazan la vida toman prioridad, pero cuenta aproximadamente con el $10 \%$ de todos los traumas vistos en una sala de emergencias ${ }^{3}$. Existen estudios que sugieren que el trauma ureteral representa menos del 1\% de todos los traumas genitourinarios, de estos el $95 \%$ de los traumas obedecen a trauma penetrante y menos del $5 \%$ son debidos a trauma contuso ${ }^{4}$. Favorecido por su especial localización anatómica, el uréter es poco vulnerable a agresiones externas por lo que en la mayoría de las ocasiones si el daño ocurre, deriva de trauma severo resultando asociado a otras lesiones intra abdominales ${ }^{5}$. El diagnóstico temprano de trauma ureteral ha mostrado ser complejo; es preciso tener un alto índice de sospecha para reducir la incidencia de complicaciones y realizar una evaluación adecuada en el tiempo ${ }^{6,7}$.

\section{Casos clínicos}

Se identificaron 5 casos de trauma ureteral en nuestro hospital durante el período septiembre de 2012 a agosto de 2013. La mediana de edad fue 24,5 años (15-46 años). En relación a la raza de los pacientes se encontró que la mayoría fueron mestizos (3 pacientes) seguidos de afro descendientes (2 pacientes).

En el aspecto educativo se encontró que los 5 pacientes sólo habían cursado estudios de secundaria.

Todos tenían estrato socioeconómico bajo y sin antecedentes patológicos. Todos tuvieron trauma penetrante.

Se manifestaron con hematuria macroscópica dos casos, un caso con dolor abdominal agudo y dos con choque hipovolémico, estos se diagnosticaron en su mayoría en las primeras $6 \mathrm{~h}$, con un caso diagnosticado de forma tardía luego de $48 \mathrm{~h}$.

Estas lesiones se encontraron principalmente localizadas hacia uréter izquierdo (4 casos) y en menor proporción en uréter derecho (1 caso), no se identificaron lesiones bilaterales.

Dado que la mayoría de las veces su manifestación clínica requirió realización de procedimiento quirúrgico, se encontró que en 4 pacientes el diagnóstico fue por exploración quirúrgica y un caso fue realizado a través de escanografía.

Las lesiones de los cinco pacientes fueron secundarias a arma de fuego; 4 pacientes tuvieron lesiones de víscera hueca asociadas y dos pacientes tuvieron lesiones vesicales asociadas.

Frente al tratamiento realizado a estas lesiones de uréter, en 3 casos se realizó uretero-ureterostomía, 1 caso se manejó de forma endoscópica con colocación de catéter de autoretención y en 1 caso se efectuó reimplante ureterovesical.

De estas intervenciones ninguna registró complicaciones (Clavien-Dindo), todos ellos se encuentran asintomáticos, fueron realizadas por un docente en un caso, otro caso manejado por un residente de primer año, un caso por residente de segundo año y dos casos manejados por residente de cuarto año. En todos los casos, los residentes actuaron bajo supervisión de un docente.

\section{Discusión}

La mayoría de los daños al uréter son causados por trauma penetrante siendo estos relacionados con heridas por arma de fuego, pudiendo éstas causar daño tanto por transección directa o dadas por la onda expansiva que se genera ${ }^{5,9}$. Acorde a lo anterior, se debe tener siempre un alto índice de sospecha tratándose de lesiones que pueden fácilmente pasar desapercibidas. La visualización directa de la lesión es la mejor forma de realizar el diagnóstico; hecho que coincide con lo encontrado en nuestros pacientes en los cuales la exploración quirúrgica fue la principal y más frecuente forma diagnóstica ${ }^{5,8,9}$.

La mayoría de las veces, las lesiones ureterales están asociadas a heridas de víscera hueca, semejante a lo encontrado en el presente artículo. Teniendo en cuenta que el trauma genitourinario es poco frecuente, el conocimiento de las lesiones asociadas incrementa la sospecha diagnóstica y así la búsqueda de lesiones ureterales para disminuir las complicaciones asociadas ${ }^{10}$.

Aunque el trauma ureteral muy raramente representa una condición que amenace la vida, si no es identificado o inapropiadamente manejado, puede llevar a importantes complicaciones, incluyendo urinoma, absceso, estrechez ureteral, fistula urinaria y potencial pérdida de la unidad renal afectada ${ }^{11}$. Por otro lado, estudios resaltan que el tipo de complicación más frecuente (en hasta el $50 \%$ de los casos) como resultado del trauma y su intervención, es la formación de fistula urinaria con formación secundaria de urinoma y sepsis ${ }^{12,13}$. No se encuentran datos en la literatura sobre diferencias en los resultados 
de las intervenciones llevadas a cabo por personal en entrenamiento o aquel con experiencia en este campo.

\section{Agradecimientos}

Agradecemos especialmente a las estudiantes de medicina y cirugía de la Universidad del Valle, Victoria Soto y Erika Barney, quienes nos apoyaron en la recolección de los datos.

\section{Referencias}

1. Correa Serna L. Guías para manejo de urgencias. tercera. Bogota, Colombia: Ministerio de la Protección Social; 2009.

2. Scalea T, Boswell S, Bonny J. Abdominal Trauma. In: Tintinalli J, Stapczynski S, Cydulka R, editors. Tintinalli's Emergency Medicine: A Comprehensive Study Guide. Seventh. China: McGraw-Hill; 2011.

3. Pfitzenmaier J, Gilfrich C, Haferkamp A, Hohenfellner M. Trauma of the ureter. Emergencies Urol. 2007;15:233-45.

4. Pereira M, Ogilvie P, Gómez R, Ryan M, Peña D. A review of ureteral injuries after external trauma. Scand J Trauma Resusc Emerg Med. 2010;18:6. doi:
10.1186/1757-7241-18-6.

5. Siram S, Gerald S, Greene W. Ureteral trauma: patterns and mechanisms of injury of an uncommon condition. Am J Surg. 2010;199:566-70.

6. Doucet J, Hoyt D. Penetrating Genitourinary Trauma. In: Brooks A, editor. Ryan's Ballistic Trauma. First. London: Springer-Verlag; 2011. p. 461-81.

7. Lee W, Lin H, Cheng T. Ureteral injury due to blunt abdominal trauma. Eur J Emerg Med. 2006;13:244-6.

8. Eshghi F, Schutte H. Value of proximal diversion and ureteral stenting in management of penetrating ureteral trauma. Urology 1988;32:99-102.

9. Summertom D, Djakovic N, Kitrey N, Kuehhas F, Lumen N, Serafetinidis E. Guidelines on urological trauma [Internet]. Europe; 2014. Available from: http://www. uroweb.org/gls/pdf/24 Urological Trauma_LR.pdf

10. Brandes S, Coburn M, Armenakas N, McAninch J. Diagnosis and management of ureteric injury: an evidence-based analysis. BJU Int. 2004;94:277-89.

11. Best $\mathrm{C}$, Petrone P. traumatic ureteral injuries: a single institution experience validating the american association for the surgery of trauma-organ injury scale grading scale. J Urol. 2005; 173:1202-5.

12. Fraga G, Borges G, Mantovani M. Penetrating Ureteral Trauma. Int Braz J Urol. 2007;33:142-50.

13. Elliott $\mathrm{S}, \mathrm{McAninch} \mathrm{J}$. Ureteral injuries from external violence: the 25-year experience at san francisco general hospital. J Urol. 2003;170:1213-6. 